\title{
Motivos de la inactividad física infantil: Una visión de niños, padres y entrenadores
}

Tellez Vasquez, Mydori Harumi; Betancourt Ocampo, Diana; Jaimes Reyes, Ana Laura; Rubio Sosa, Héctor Igor; González González, Alejandro

Motivos de la inactividad física infantil: Una visión de niños, padres y entrenadores

MHSalud, vol. 18, núm. 2, 2021

Universidad Nacional, Costa Rica

Disponible en: https://www.redalyc.org/articulo.oa?id=237066090003

DOI: https://doi.org/10.15359/mhs.18-2.3

\section{(c) $($ ) $\Theta \Theta$}

Esta obra está bajo una Licencia Creative Commons Atribución-NoComercial-SinDerivar 3.0 Internacional. 


\section{Motivos de la inactividad física infantil: Una visión de niños, padres y entrenadores}

Reasons for Child Physical Inactivity: A View from Children, Parents, and Coaches

Razões para a inatividade física infantil: Uma visão das crianças, pais e treinadores

Mydori Harumi Tellez Vasquez

Universidad Anábuac, México

mydoritellez@gmail.com

(iD https://orcid.org/0000-0002-5506-7208

Diana Betancourt Ocampo

Universidad Anábuac, México

diana.betancourt@anahuac.mx

iD https://orcid.org/0000-0001-6405-9827

Ana Laura Jaimes Reyes

Universidad Anábuac, México

laura93jr@gmail.com

(iD https://orcid.org/0000-0002-5431-0739

Héctor Igor Rubio Sosa

Universidad Anábuac, México

igor.rubio@anahuac.mx

(D) https://orcid.org/0000-0003-1213-9061

Alejandro González González

Universidad Anábuac, México

alejandro.gonzalezg@anahuac.mx

(D) https://orcid.org/0000-0002-6323-3851
DOI: https://doi.org/10.15359/mhs.18-2.3

Redalyc: https://www.redalyc.org/articulo.oa? $\mathrm{id}=237066090003$

Recepción: 11 Mayo 2020

Aprobación: 29 Enero 2021

\section{Resumen:}

La realización de actividad física desde la infancia y a lo largo de la vida adulta, permite que se mantenga un perfil de riesgo bajo respecto a numerosos padecimientos. El objetivo de este estudio fue identificar las dificultades que generan la inactividad física en niños, para lo cual se contó con una muestra de 2,621 niños y niñas de 13 ciudades del país, 3,754 padres de estos niños, y 134 entrenadores de diferentes escuelas presentes en la República Mexicana. Para obtener la información de esta población, se utilizó un cuestionario de 17 preguntas abiertas y cerradas, el cual fue una adaptación del instrumento: State of Play: Youth Sports Survey (The Aspen Institute, 2018), mientras que a los padres y madres de familia se les preguntó cuáles consideraban que podrían ser las principales dificultades que enfrentaban para fomentar la actividad física y/o deportiva en sus hijos e hijas, finalmente a sus entrenadores se les preguntaron las razones principales por la que los niños no practican deportes o actividad física. Los resultados mostraron que, en la muestra general de niños, el aspecto principal que genera su inactividad física se relaciona con el cumplimiento de responsabilidades; los padres mencionaron como causa principal la falta de tiempo, y los entrenadores el mal uso de la tecnología. Palabras Clave: actividad física, inactividad física, niñez, motivos.

\section{Abstract:}

Physical activity from childhood and throughout adult life allows a low-risk profile to be maintained compared to many conditions. This study aimed to identify the difficulties that lead to physical inactivity in children. For the study, there was a sample of 2,621 children from 13 cities in the country, 3,754 parents of these children, and 134 coaches from different schools in the Mexican Republic. A questionnaire of 17 open and closed questions was used to obtain children's information; the questionnaire was an adaptation of the instrument State of Play: Youth Sports Survey (The Aspen Institute, 2018). Parents were asked what they felt 
might be the main challenges they had in fostering physical and/or sporting activity in their children. Finally, coaches were asked the main reasons why children do not play sports or physical activity. In the general sample of children, the results showed that the main aspect of their physical inactivity relates to responsibilities; parents mentioned, as the main cause, the lack of time, and coaches reported misused the technology.

KEYWORDS: physical activity, physical inactivity, children, reasons.

\section{Resumo:}

A realização de atividade física desde a infância e ao longo da vida adulta, permite que seja mantido um perfil de baixo risco em relação a muitas doenças. O objetivo deste estudo foi identificar as dificuldades que a inatividade física gera nas crianças, para a qual houve uma amostra de 2.621 crianças de 13 cidades do país, 3.754 eram os pais dessas crianças, e 134 eram treinadores de diferentes escolas presentes na República Mexicana. Para a obtenção das informações das crianças, foi utilizado um questionário com 17 questões abertas e fechadas, que foi uma adaptação do instrumento: Estado do jogo: Youth Sports Survey (The Aspen Institute, 2018), enquanto os pais eram questionados sobre o que eles consideravam ser as principais dificuldades que enfrentavam para promover a atividade física e/ou esportiva entre seus filhos, e finalmente, os treinadores foram questionados sobre os principais motivos pelos quais as crianças não participam de esportes ou atividade física. Os resultados mostraram que, na amostra geral de crianças, o principal aspecto que gera sua inatividade física está relacionado ao cumprimento de responsabilidades; os pais citaram a falta de tempo como a principal causa e o uso indevido da tecnologia pelos treinadores.

PalaVras-CHAVE: atividade física, sedentarismo, crianças, motivos.

\section{INTRODUCCIÓN}

Según la Organización Mundial de la Salud (OMS) (2019a, 2019b), la actividad física es todo aquel movimiento corporal producido por los músculos esqueléticos que exige un gasto de energía. No realizar este tipo de actividad es considerado el cuarto factor de riesgo de mortalidad mundial, además de que la falta de esta es considerada la causa principal del $27 \%$ de los casos de diabetes y casi el $30 \%$ de la carga de cardiopatía isquémica. La actividad física comprende al ejercicio, pero no debe confundirse con él, ya que esta considera también otras actividades relacionadas con movimiento corporal que se realizan tanto en el juego como en el trabajo, medios de transporte, tareas domésticas, etc.

En el 2016, la OMS (World Health Organization, 2016) estimó que, a nivel mundial, solo el $19.3 \%$ de la población infantil y adolescente era físicamente activa, lo cual resulta sumamente preocupante, pues si se considera que existe evidencia que vincula los patrones de actividad física en este tipo de poblaciones con los patrones que se muestran presentes en la etapa adulta; es decir, quienes no realizan actividad física en etapas tempranas del desarrollo, poseen mayor probabilidad de presentar enfermedades crónicas no transmisibles, como accidentes cerebrovasculares, diabetes y cáncer (Pietilainen et al., 2008).

Ante esta problemática, la OMS (Organización Mundial de la Salud, 2004c) ha sugerido mantener un monitoreo a nivel mundial sobre los niveles de actividad física con la finalidad de obtener datos que permitan valorar la efectividad de las políticas y programas que se realizan en cada país con respecto a la promoción de la activación física. No obstante, en nuestro país se carece de información sobre la prevalencia y tendencia de actividad física en la población infantil (The Global Observatory for Physical Activity-GoPA, 2016).

El papel que juega la actividad física en el desarrollo del niño y la niña es un aspecto determinante en cuestiones de salud, bienestar y capacidad de aprendizaje durante toda su vida, por lo que tanto la familia como el contexto escolar son factores que influyen como motivadores o, en otros casos, como barreras. Por un lado, la familia es el ambiente en el que los niños pasan una mayor parte del tiempo; son los miembros de esta enseñan usos y costumbres y de quienes los y las infantes aprenden sus primeros comportamientos interpersonales, por cuanto la familia cumple con el rol de brindar los primeros modelos fundamentales para el desarrollo socioafectivo del niño; son los miembros de cada familia los que brindan guías de comportamiento y vínculos emocionales, creencias y valores a la población infantil. 
Por otro lado, la escuela significa para el niño o la niña el medio en el cual conocen a sus pares, esas personas de su edad con las que comparten creencias, aprenden nuevas formas de solucionar problemas, pero también es el espacio escolar donde conviven con otros adultos, los cuales, a pesar de que sus actividades se enfocan en la enseñanza de las distintas materias curriculares, muchas veces también influyen, por su manera de ser y enseñar, en la motivación de los niños y las niñas en aspectos relacionados con el ámbito deportivo, las artes, etc. (Arón y Milicic, 1999a; Arón y Milicic, 1999b; Bronfenbrenner, 1979, 1989; Vigotsky, 1979).

Uno de estos tantos aspectos que se les inculcan a los niños y las niñas desde una edad temprana, tanto en el ambiente escolar como familiar, es la actividad física, la cual tiene considerables beneficios para la salud de cada individuo. Algunos de estos beneficios se relacionan con: reducir el riesgo de hipertensión, accidentes cerebrovasculares y diabetes; la mejora de la salud ósea, la salud funcional, el estado muscular y el estado cardiorrespiratorio; el equilibrio energético y el control de peso (Organización Mundial de la Salud, 2018).

Una mayor realización de actividad física desde la infancia, y a lo largo de la vida adulta, permite que se mantenga un perfil de riesgo bajo, así como menores tasas de morbilidad y mortalidad relacionadas con enfermedades cardiovasculares y diabetes mellitus. También se ha observado que la práctica de actividad física, ya sea moderada o vigorosa, se relaciona positivamente con la fuerza muscular, mejorando considerablemente el fortalecimiento muscular en niños y jóvenes que la realizan dos o tres veces por semana; este tipo de actividades no necesariamente deben ser planificadas, pues pueden tratarse de actividades espontáneas que se presentan mediante el juego, realizando movimientos de empuje y tracción. Asimismo, las actividades como saltos, volteretas, carreras y similares. propician un esfuerzo óseo que genera una mejora en la densidad de los huesos de los niños y jóvenes (Organización Mundial de la Salud, 2010).

Matsudo (2012) menciona algunos otros beneficios que se obtienen de realizar actividad física en la fase escolar, tanto en niños como en adolescentes; estos son: el mejoramiento de la relación con los padres, la disminución de la delincuencia y/o reincidencia, la disminución del consumo de alcohol y el aumento de la abstinencia, así como la reducción de alteraciones del comportamiento, el mejoramiento de la responsabilidad de los jóvenes y el incremento del desempeño académico y vocacional.

Existen estudios, tales como el de Bornstein et al. (2011), que muestran que actualmente la actividad física realizada por la población infantil y adolescente no cumple con los estándares establecidos por la OMS. En México, la Encuesta Nacional de Salud y Nutrición (ENSANUT) en el año 2012 (Gutiérrez, et al., 2012), mostró que del total de niños y niñas de entre 10 y 14 años participantes del estudio, el 82.8 \% (87.3 \%mujeres, y $78.2 \%$ hombres) no cumplía con las recomendaciones de la OMS en referencia a la actividad física, y se consideraban como inactivos. Por su parte, en el año 2016 incrementó la participación de la población infantil en estas actividades, ya que tan solo el $39 \%$ de la muestra total se consideraba como inactiva (48.5\%de las mujeres y $29.6 \%$ de los hombres); esto, a pesar de ser un dato favorable, casi la mitad de las mujeres de la muestra total y una quinta parte de la proporción de hombres siguen sin realizar los niveles adecuados de actividad física, lo cual sigue siendo una cifra de preocupación considerando las repercusiones que esto puede traer, tanto en la niñez como en la vida adulta (Hernández, et al., 2016). Finalmente, en el año 2018, la misma encuesta únicamente reportó resultados para la población de 20 a 69 años de edad, la cual señaló que tanto la muestra total como la comparación entre hombres y mujeres, reportó en mayor medida el realizar menos de 150 minutos de actividad física por semana (Instituto Nacional de Salud Pública y Instituto Nacional de Estadística y Geografía, 2018).

Cabe resaltar que muchas personas no realizan la suficiente actividad física o mantienen una vida sedentaria debido a diversos obstáculos que lo impiden o restringen; según la American Academy of Pediatrics (2016), algunas de estas barreras son: la falta de tiempo, el acceso a deportes de interés, la autopercepción del individuo a la hora de evaluarse capaz o apto para practicar un deporte, la inseguridad que presentan algunos de los lugares que podrían considerase apropiado para practicar actividad física, la condición física de cada persona, entre otros aspectos. 
Schmutz et al. (2017), estudiaron una muestra de 476 niños y niñas de edades entre dos y seis años que identificaron los factores ambientales (seguridad del área de residencia, el acceso a espacios adecuados para realizar este tipo de actividades, etc.) como factores que presentan mayor influencia en el comportamiento sedentario, mientras que otros aspectos personales (Autorregulación, temperamento, etc.) fueron señalados como factores de influencia positiva en la práctica de actividad física.

Ante lo anterior, resulta importante señalar que sedentarismo e inactividad física son conceptos diferentes; por un lado, el sedentarismo es la falta de movimiento durante las horas de vigilia, este se relaciona generalmente con práctica de actividades que sobrepasan el gasto energético basal como ver televisión, estar acostado o sentado por largos periodos, mientras que la inactividad física se encuentra relacionada con el incumplimiento de las recomendaciones mínimas internacionales referentes a la práctica de actividad física (Cristi-Montero et al., 2015). Por otro lado, Muñoz-Daw et al. (2016) reportan que el $35.1 \%$ de las niñas y $22.5 \%$ de los niños, de los cuales obtuvieron información, no realizaba actividad física, debido principalmente a la desmotivación (para las niñas) y la falta de tiempo (para los niños).

Por otro lado, diversos autores (Granda et al., 2010; Martínez et al., 2010; Portela \& López, 2017; Serra et al., 2010) encontraron que los motivos por los cuales los niños y las niñas no realizan actividad física son: las obligaciones escolares y del hogar, la falta de tiempo y la pereza o fatiga. Sin embargo, también se encontró que un $90 \%$ de los niños de la muestra estudiada dijeron que el hecho de no realizar actividad física no se encontraba relacionada con la ausencia de la práctica en sus familiares; asimismo, el $89 \%$ de la muestra mencionó que esta inactividad resultaba independiente al hecho de que sus amigos practiquen o no alguna actividad física.

Otros aspectos encontrados en la literatura resaltan factores que determinan que los niños y las niñas no realicen actividad física: la falta de apoyo social, la intimidación por parte de sus compañeros, el uso de artículos electrónicos en la escuela, el poco acceso a lugares adecuados para hacer actividad física, no tener a disposición a otra persona con quien practicar, la inseguridad, miedo a lesionarse, entre otros aspectos (Álvarez, 2016; Serra, et al., 2010).

Respecto a los motivos por los cuales los padres de familia consideran que los niños no realizan actividad física, Fernández-Argüelles y Fernández-Río (2018) y Yamamoto -Kimura et al. (2013) resaltan que la falta de tiempo, la falta de interés por algún deporte, la falta de tiempo de los padres, la falta de recursos (monetarios y físicos), la distancia que hay entre el hogar y los espacios dedicados a este tipo de actividades (deportivos, albercas, etc.), así como el desconocimiento de los lugares a los cuales podrían llevar a sus hijos para ejercitarse, resultan ser razones primordiales por las cuales la población infantil no realiza actividad física, según la percepción de los padres.

Como se observa, la situación actual respecto a la activación física en población infantil en nuestro país resulta alarmante; esto aunado a los datos que presenta la ENSANUT (Encuesta Nacional de Salud y Nutrición, 2016) en cuanto a que el $33.2 \%$ de la población infantil entre 5 y 11 años en México sufren sobrepeso $(17.9 \%)$ y obesidad $(15.3 \%)$ vuelve más complicado el panorama. De acuerdo con datos del Observatorio Global de Obesidad (World Obesity Federation, 2019), México ocupa el segundo lugar de obesidad infantil en la región de las Américas. Considerando que la actividad física es el único componente del gasto de energía que puede modificarse voluntariamente, se debe considerar su práctica como un elemento fundamental para incidir en el problema de peso poco saludable presente en población infantil.

Aunado a la información anterior, es importante señalar que existen numerosos estudios que dan cuenta de los distintos beneficios que conlleva realizar actividad física a edades tempranas, entre los cuales se pueden mencionar: un mejor desarrollo motor, equilibrio y flexibilidad (Roberts, 2000), mayor resistencia ósea (Laing, et al., 2002), mayor autoestima y reducción de sintomatología depresiva (Haugland, et al. 2003), así como la formación de hábitos de ejercicio durante toda la vida (Twisk, et al., 2000). Además, no se debe olvidar que durante los primeros años de vida resulta ser un momento crucial dentro del desarrollo de cada individuo, pues en esta etapa se moldean tanto cogniciones como conductas. 
Debido a la información mostrada hasta este punto, la presente investigación se planteó como objetivo identificar las dificultades que generan la inactividad física en una muestra de población infantil mexicana, considerando a su vez las dificultades que perciben los padres de familia de estos niños y niñas, y los profesores de educación física de diversas entidades de la República Mexicana, pues, como se mencionó con anterioridad, tanto el aspecto familiar como los entrenadores deportivos en el contexto escolar fungen como motivadores y ejemplos de la práctica de estas actividades, mostrándose como modelos que, en muchos casos, afectan de manera exponencial a los niños y niñas, generando en ellos el interés y dedicación necesaria para mantener una vida saludable. Finalmente se espera que dicha información pueda servir de base para un modelo de intervención educativa en cuestiones de salud y actividad física en el ámbito escolar y familiar.

\section{MÉTodo}

Se llevo a cabo un estudio no experimental, transversal, descriptivo.

\section{Participantes}

Se seleccionó una muestra no probabilística de 2621 niños y niñas (47.1\% hombres y $52.5 \%$ mujeres) de 13 localidades del país (Acapulco, Aguascalientes, Chalco, Conkal, Guadalajara, León, León Villas, Lerma, Monterrey, Morelia, Puebla, Tijuana, y Torreón) pertenecientes a un grupo de escuelas privadas (nivel socioeconómico medio-bajo), con un rango de edad de 8 a 13 años $(M=9.86$, D.E.=1.24), quienes cursaban de tercero a sexto grado de educación primaria $\left(22.8 \%\right.$ de $3^{\circ}, 25.2 \%$ de $4^{\circ}, 26.1 \%$ de $5^{\circ}$, y $25.6 \%$ de $\left.6^{a}\right)$, la mayoría (80.9\%) informó vivir con ambos padres.

Además, se obtuvo información de los padres y madres de los niños participantes, los cuales dieron un total de 3,754 (44\% hombres y $56 \%$ mujeres), con un promedio de edad de 38.91 años (D.E.=6.86). El 74.2 \%de estos indicó que se encontraban casados y la mayoría (75.8 \%) informó que vivían con su pareja e hijos. Respecto a su escolaridad, el mayor porcentaje de los padres $(34.3 \%)$ indicó que tenía estudios de nivel de licenciatura, seguido de quienes mencionaron que tenían estudios de nivel bachillerato $(24.7 \%)$ y, en tercer lugar, con el $18.4 \%$ los padres con nivel secundaria. El $59.3 \%$ de los padres y madres encuestados informó que tienen trabajo de tiempo completo, $22.3 \%$ mencionaron que se dedican al hogar y, con un $11.1 \%$, los que reportaron tener un trabajo de medio tiempo. En el estudio también participaron 134 profesionales en educación física, de los cuales $64.2 \%$ fueron hombres y $35.8 \%$ mujeres, con un promedio de edad de 37.29 años (D.E.=6.86).

\section{Instrumentos}

Con el objetivo de obtener información de la población infantil, se diseñó un cuestionario que contenía un apartado de datos sociodemográficos (sexo, edad, estructura familiar, grado en curso), así como un indicador de la Encuesta Nacional de Salud y Nutrición (Instituto Nacional de Salud Pública y Instituto Nacional de Estadística y Geografía, 2018) que permite evaluar el número de días que los menores estuvieron activos al menos 60 minutos por día, para poder ubicarlos de acuerdo con los lineamientos establecidos por la Organización Mundial de la Salud (2010) como niños físicamente activos o no activos. Además, como parte del cuestionario, se incluyó una pregunta donde se les pedía a la población infantil encuestada que indicara cuáles eran las razones por las que no practicaban deportes o no lo hacían regularmente; esta fue una pregunta cerrada con 12 opciones de posibles respuestas, donde los menores podían marcar todas las opciones que considerarán posibles (presencia/ausencia), dicha pregunta fue adaptada de State of Play: Youth Sports Survey (The Aspen Institute, 2018), cuestionario que valora distintos aspectos de la actividad física y 
deportiva (p.e., tipos de deportes y actividades físicas que practican o les gustaría practicar a los niños, lugares donde suelen practicarlos, medios de transporte para acudir a los espacios donde practican, etc.).

Por lo que respecta a los padres y madres, se diseñó un cuestionario que incluía un apartado de datos sociodemográficos (sexo, edad, estructura familiar, grado de estudios, estado civil, ocupación) y una pregunta abierta sobre las principales dificultades que enfrentaban para fomentar la actividad física y/o deportiva en sus hijos. En cuanto al instrumento utilizado con la población profesional en educación física, también se diseñó para el presente estudio un breve cuestionario con un apartado sociodemográfico (sexo, edad, formación como entrenador o profesor de educación física) y una pregunta abierta donde se les cuestionó sobre las principales razones por las que los niños y niñas, según su opinión, no practican deportes o actividad física.

\section{Procedimiento}

En cuanto a la aplicación de los instrumentos con la población infantil, padres y madres de familia, inicialmente se contactó a las autoridades de las instituciones educativas para invitarlos a participar en el estudio, se les explicó cuál era el objetivo general de estudio y se describió la forma de aplicación de los instrumentos. Posteriormente, se les envío a los padres y madres de familia un consentimiento informado tanto para su posible participación como para la colaboración de los menores. Los padres y madres que firmaron el consentimiento informado, tanto de su participación como la de los niños y niñas, fueron a los que se les envió, mediante un sobre cerrado, los cuestionarios respectivos para el padre y la madre. Una vez resueltos, ellos tenían que regresarlos al colegio, donde los profesores y profesoras de cada grupo participantes se encargaban de archivarlo.

En el caso de los niños y niñas, la aplicación se realizó dentro de los salones de clase, la cual se llevó a cabo de manera grupal por parte de las psicólogas de la institución educativa. Para los niños de $3^{\circ}$ y $4^{\circ}$ grado, las psicólogas en cuestión fueron las encargadas de leer cada una de las preguntas del cuestionario, mientras la población estudiantil iba respondiendo; en el caso de los niños y niñas más grandes $\left(5^{\circ}\right.$ y $6^{\circ}$ grado), las psicólogas leyeron las instrucciones a todo el grupo y, posteriormente, cada uno de los niños y cada una de las niñas fueron respondiendo el cuestionario de manera autoaplicable. El levantamiento de información, tanto de padres como de niños, se realizó en aproximadamente dos semanas para todos los colegios participantes.

Por lo que respecta al grupo de entrenadores y/o profesores de educación física, se contactó al coordinador general de un grupo de escuelas privadas, a quien se le explicó el propósito del estudio y quien accedió a enviar el instrumento a sus entrenadores y profesores de educación física para su posible participación. El cuestionario se realizó en formato electrónico (Formulario de Google) y fue compartido por el coordinador a su grupo de profesores y entrenadores de educación física. Es importante señalar que antes de poder responder las preguntas del cuestionario, los profesionales de educación física tenían que indicar (dentro del formato electrónico) que aceptaban participar en el estudio (consentimiento informado).

\section{Análisis de datos}

Un primer análisis que se realizó se centró en obtener las frecuencias relativas respecto a la variable de actividad física de los niños(as). Posteriormente, con las respuestas brindadas por los y las menores respecto a las razones para no realizar actividad física o no hacerlo de manera frecuente se obtuvieron tablas de contingencia comparando la frecuencia de ocurrencia por el sexo de esta población, y se calcularon los coeficientes de Ji cuadrada de Pearson.

Para las preguntas abiertas de los padres y las madres de familia, así como de las personas profesionales en educación física, se realizó análisis de contenido, cuyo propósito fue la identificación de determinados elementos y componentes de los documentos escritos: letras, silabas, frases, párrafos, etc., y su clasificación 
bajo la forma de variables y categorías para la explicación de los fenómenos investigados; afín al objetivo de estudio de esta investigación se buscaban las razones y/o dificultades frente a la práctica deportiva de los niños y niñas.

La construcción de estas categorías siguió los criterios fundamentales para que fueran pertinentes, exhaustivas, homogéneas y mutuamente excluyentes (Duverger, 1986, p.181). Ya con las categorías elaboradas, se realizó una tabla de contingencia donde se compararon las respuestas por informante: madres, padres y entrenadores(as) y se obtuvo el coeficiente de Ji cuadrada de Pearson para determinar si había una relación significativa por el tipo de categoría respecto al informante.

Finalmente, se compararon las categorías sobre las razones que mencionaron los padres y madres de familia para no realizar actividad física de acuerdo con la clasificación de actividad física del menor, esto se realizó por medio de una tabla de contingencia donde se obtuvo el estadístico de Ji cuadrada de Pearson.

\section{Resultados}

En un primer momento, de acuerdo con los lineamientos establecidos por la Organización Mundial de la Salud (2010), se clasificó a los menores en niños activos/niñas activas o niños no activos/niñas no activas físicamente; los resultados de esta clasificación mostraron que la muestra estudiada el $82.6 \%$ pudo ubicarse como niños y niñas físicamente no activos y el $17.4 \%$ fueron niñas y niños activos físicamente. Para analizar las razones que mencionaron los niños y niñas para no practicar deportes o no hacerlo de forma regular solo se tomó a la muestra que se clasificó como no activos.

Los resultados mostraron que en seis de las categorías se encontró una relación significativa con respecto al sexo de los y las menores, donde en las categorías de confianza, contexto y falta de apoyo parental, fue un mayor número de niñas que de niños los que reportaron estas razones para no hacer deporte. En las categorías de falta de gusto o interés, malas experiencias y salud, fue significativamente mayor el número de niños que indicaron que estas eran algunas de las razones por las cuales no hacían deporte. Por lo que se refiere a la categoría de responsabilidades, si bien en esta no se encontró una relación significativa por sexo, fue la categoría más elegida por la mayoría de niños y niñas (Tabla 1). 
MHSALUD, ISSN: 1659-097X, 18(2), JULIO-DiciemBre, 2021, PP 1-14

Tellez Vasquez, Betancourt Ocampo, Jaimes Reyes, Rubio Sosa, González González

TABLA 1

Razones por las que los niños y niñas no practican actividad física y/o deporte

\begin{tabular}{lccccc}
\hline \multirow{2}{*}{\multicolumn{1}{c}{ Razón }} & Niños & Niñas & & \multirow{2}{*}{$\mathbf{X}^{\mathbf{2}}$} & $\boldsymbol{p}$ \\
\cline { 2 - 3 } & $\mathbf{\%}$ & $\mathbf{\%}$ & & .008 \\
\hline Confianza & 27.2 & 32.7 & & 6.97 & .001 \\
Contexto & 11.8 & 17.2 & & 11.49 & .745 \\
Economía & 15.0 & 14.5 & & 0.10 & .018 \\
Falta de apoyo parental & 1.2 & 2.7 & & 5.60 & .016 \\
Falta de gusto o interés & 14.9 & 11.3 & & 5.75 & .746 \\
Falta de opciones & 14.4 & 15.0 & & 0.10 & .200 \\
Falta de posibilidades & 13.7 & 11.7 & & 1.64 & .439 \\
Falta de tiempo & 2.6 & 2.1 & & 0.59 & .006 \\
Malas experiencias & 16.2 & 11.9 & 7.50 & .813 \\
Responsabilidades & 43.2 & 43.8 & 0.05 & .001 \\
Salud & 18.9 & 13.2 & 12.10 & .548 \\
Tecnología & 0.3 & 0.2 & 0.36 & \\
\hline
\end{tabular}

Nota: Porcentajes correspondientes a la muestra que reportó no realizar actividad física.

En la Tabla 2 se presentan los resultados sobre las dificultades que presentan tanto padres y madres de familia, así como entrenadores(as), para fomentar la actividad física en niños y niñas. Los hallazgos mostraron una relación significativa $\left(X^{2}=557.16, \mathrm{p}<001\right)$. En el caso de los padres y madres la categoría que se reportó con mayor frecuencia fueron aspectos relacionados a la falta de tiempo y el exceso de trabajo, tanto de ellos como de las actividades escolares de sus hijos y/o hijas.

Para los sujetos profesionales en educación física, la categoría que se identificó como prioritaria fue la del uso excesivo de dispositivos electrónicos (p.e., celulares, videojuegos, televisión, computadora, etc.). En segundo lugar, para las madres se ubicó los aspectos económicos como una dificultad a la que se enfrentan para fomentar la actividad físico-deportiva en los niños y niñas; para los padres, el uso excesivo de dispositivos electrónicos (p.e., celulares, videojuegos, televisión, computadora, etc.) se encontró como la segunda categoría con mayor mención. Para el grupo de profesionales en educación física, la categoría con aspectos sobre falta de educación y hábitos, sí como poca promoción e información sobre las actividades que pueden hacer los menores se ubicó en segundo lugar.

La categoría que apareció en tercer lugar para los tres grupos de informantes fueron los factores asociados al propio contexto, que incluyen aspectos como inseguridad en los espacios donde se pueden realizar actividades físico-deportivas, falta de lugares adecuados, falta de accesibilidad a ese tipo de espacios, así como cuestiones de distancia y problemas de transporte para poder llegar a esos lugares. 
MHSALUD, ISSN: 1659-097X, 18(2), JULIO-DiCIEMBRE, 2021, PP 1-14 Tellez Vasquez, Betancourt Ocampo, Jaimes Reyes, Rubio Sosa, González González

TABLA 2

Dificultades para fomentar la actividad fisica en niños y niñas por informante

\begin{tabular}{lccc}
\hline & \multicolumn{3}{c}{ Informante } \\
\cline { 2 - 4 } & Madre & Padre & Entrenador \\
\cline { 2 - 4 } & $\%$ & $\%$ & $\%$ \\
\hline Dificultades de tiempo y responsabilidades & 59.3 & 60.7 & 8.0 \\
Problemas económicos & 10.1 & 6.1 & 7.4 \\
Uso excesivo de dispositivos electrónicos & 6.4 & 8.7 & 21.0 \\
Factores del contexto & 8.7 & 7.8 & 14.8 \\
Falta de educación, hábitos, promoción e información & 2.5 & 2.9 & 8.2 \\
Desinterés, falta de motivación y flojera & 8.1 & 7.5 & 13.6 \\
Falta de adultos como modelos y promotores de & 3.8 & 5.0 & 1.1 \\
conductas saludables & 1.1 & 1.3 & 8.0 \\
Factores asociados a salud/enfermedad & - & - & \\
Aspectos relacionados con el ámbito educativo & & & \\
\hline
\end{tabular}

Por otro lado, se analizaron las dificultades que enfrentan los padres y las madres para fomentar la actividad física con hijos que son físicamente activos y no activos. Como se puede observar en la Tabla 3, tanto para las madres como para los padres, las dificultades más frecuentes fueron aquellas relacionadas con la falta de tiempo y exceso de tareas (tanto de los padres/madres como de los niños/niñas), esto sin importar si su hijo o hija es o no activo físicamente. El uso excesivo de dispositivos electrónicos, así como la falta de interés para realizar actividades físico-deportivas, la falta de motivación y la flojera fueron las siguientes dos categorías que se mencionaron con más frecuencia, tanto de padres como de madres sin importar si sus hijos(as) eran o no físicamente activos. Cabe señalar que no se encontró una relación significativa ni para lo reportado por las madres $\left(X^{2}=5.44, \mathrm{p}>05\right)$ ni por los padres $\left(X^{2}=3.57, \mathrm{p}>05\right)$.

TABLA 3

Dificultades para fomentar la actividad física en niños y niñas por clasificación de su actividad fisica

\begin{tabular}{lcc}
\hline & \multicolumn{1}{c}{$\begin{array}{c}\text { Niños no activos } \\
\text { físicamente }\end{array}$} & $\begin{array}{c}\text { Niños activos } \\
\text { físicamente }\end{array}$ \\
\cline { 2 - 3 } & $\%$ & 75.0 \\
\hline Dificultades de tiempo y responsabilidades & 73.8 & 4.9 \\
Desinterés, falta de motivación y flojera & 6.0 & 6.6 \\
Uso excesivo de dispositivos electrónicos & 5.3 & 4.9 \\
Factores del contexto & 3.8 & 3.3 \\
Problemas económicos & 4.9 & 2.6 \\
Falta de educación, hábitos, promoción e información & 2.1 & 2.0 \\
Falta de adultos como modelos y promotores de conductas & 3.5 & 0.7 \\
saludables & 0.6 & 75.2 \\
Factores asociados a salud/enfermedad & & 6.4 \\
Dificultades de tiempo y responsabilidades & 72.2 & 6.0 \\
Uso excesivo de dispositivos electrónicos & 8.3 & 4.0 \\
Desinterés, falta de motivación y flojera & 4.8 & 2.8 \\
Factores del contexto & 4.1 & 3.2 \\
Falta de adultos como modelos y promotores de conductas & 4.0 & 2.0 \\
saludables & 3.0 & 0.4 \\
Problemas económicos & 2.7 & 0.9 \\
Falta de educación, hábitos, promoción e información & & \\
Factores asociados a salud/enfermedad & & \\
\hline
\end{tabular}


MHSALUD, ISSN: 1659-097X, 18(2), JULIO-DiciEMBRE, 2021, PP 1-14

Tellez Vasquez, Betancourt Ocampo, Jaimes Reyes, Rubio Sosa, González González

\section{Discusión}

En este estudio se identificaron las razones por las cuales los niños no realizan actividad física a través de la percepción de diversos actores (niños y niñas, padres y madres de familia y profesionales en educación física), dado que no existe mucha evidencia en nuestro país acerca de lo que refieren los actores involucrados, lo cual resulta importante, si es que se quiere tener un panorama completo acerca de lo que pudiese estar ocurriendo con los niños y niñas, y por qué cada vez más se incrementa el número de aquellos que no practican algún deporte.

De acuerdo con los resultados, llama la atención que para el caso de los niños y niñas, la razón principal por la cual no practican actividad física y/o deporte en nuestro país se relaciona con las responsabilidades que estos tienen, ya sea la realización de tareas escolares, deberes en el hogar y/o otras situaciones relacionadas con esto mismo, aspectos expuestos también en investigaciones previas (Granda, et al., 2010; Portela y López, 2017); esto resulta interesante dado que se habla de niños y niñas de 8 a 12 años de edad, de quienes no se esperaría una percepción de responsabilidades asumidas muy grande, sin embargo, lo que esta población percibe es una carga de responsabilidades que pareciera ser el motivo que impide dedicarse a actividades lúdicas, lo cual favorecería el desarrollo mismo de los niños.

Un dato interesante respecto a la presente investigación trata de lo encontrado al analizar las diferencias por sexo, donde para las niñas resulta más importante la confianza, el factor del contexto y el apoyo parental, para, en su caso, decidirse a practicar deporte, lo cual coincide en cierta forma con lo reportado en otros estudios, en donde se hacía mención de la inseguridad de los lugares para practicar este tipo de actividades así como la desmotivación, la falta de apoyo social en el ambiente escolar y la intimidación por parte de sus compañeros (Álvarez, 2016; Serra et al., 2010; American Academy of Pediatrics, 2016; Muñoz-Daw et al., 2016; Schmutz, 2017), es decir, pareciera ser que, en particular, las niñas perciben un entorno poco seguro, lo que, aunado a la falta de confianza en sí mismas y a la falta de apoyo por parte de los padres principalmente, refuerza su condición de desventaja frente a la práctica deportiva.

Sin embargo, esto difiere de lo reportado por Martínez et al. (2010), quien refiere que la razón primordial de la inactividad física en niñas fue la falta de tiempo, lo que podría deberse a la diferencia de edad existente entre los participantes del presente estudio y el de los autores (mayores de 12 años), confirmando la relación que existe entre la edad y las razones argumentadas por los niños.

Por otra parte, en lo que respecta a los niños, la falta de gusto o interés, las malas experiencias y la salud, fueron las razones más mencionadas para la presencia de inactividad física en los participantes de esta investigación; al parecer, las principales razones en estos casos se encuentran enfocadas en lo individual, más que en lo contextual, esto es, aquellas que tienen que ver con la propia disposición de los niños, aunado a la existencia de malas experiencias en la práctica inicial, lo cual resulta fundamental para que un niño desee volver a intentarlo y que, regularmente, se asocian con el que los niños no alcanzan a cubrir las expectativas de los adultos en sus primeras experiencias, hecho que guarda relación con lo reportado en estudios previos (Granda et al., 2010; Martínez et al., 2010; Portela \& López, 2017; Serra et al., 2010).

De manera particular, lo reportado por los niños, relacionado con su salud, coincide con lo que la American Academy of Pediatrics (2016) señaló como una de las barreras que los niños indicaban como razones del hecho de padecer sobrepeso o que al hecho de que no se sintieran en forma para realizar dichas actividades, lo coloca en una situación frustrante, al no poder realizar ejercicio, independientemente de que lo quisieran hacer, lo cual resulta preocupante en esta etapa de su desarrollo.

Sin embargo, aspectos como las malas experiencias, la economía y la falta de opciones, fueron situaciones que no se encontraron en la literatura revisada; esto podría deberse a que dichos estudios fueron realizados en otros países y, por lo tanto, sería apropiado evaluar de manera más profunda estos elementos para poder generar mejores condiciones para los infantes y así disminuir la presencia de inactividad física en el país, trabajando a partir de las necesidades propias de la población, realizando entonces un trabajo conjunto con 
otras disciplinas, desde ingenieros, médicos, psicólogos y personas expertas en las áreas con necesidad de intervención.

Posteriormente, a través de un segundo análisis, enfocado en la contratación de los argumentos expuestos entre los tres informantes (madre, padre y persona profesional en educación física), se observó que la razón más referida por padres y madres fue la falta de tiempo disponible para poder fomentar la actividad física en sus hijos e hijas, lo cual se relaciona con un exceso de trabajo, característico de poblaciones urbanas, en donde además de tener que cumplir con las responsabilidades laborales, se requiere invertir tiempo considerable en los traslados del hogar hacia los lugares de trabajo y viceversa, argumentos expuestos también en otros estudios (Fernández-Argüelles \& Fernández-Río, 2018; Yamamoto-Kimura et al., 2013) aunado a que muchas de las veces los niños y las niñas tienen que iniciar sus tareas escolares, aún en ausencia de sus padres, realidad que trae como consecuencia una falta de vigilancia por parte de los padres y, por otra parte, que muchas veces los niños, prefieren pasar el tiempo usando dispositivos electrónicos más que dedicarse a practicar algún deporte (Álvarez, 2016; Serra, et al., 2010), lo cual representó la principal razón reportada por los entrenadores en este estudio.

No sorprende el hecho de haber encontrado los problemas económicos argumentados por los padres y las madres correspondientes, como otra de las razones que limitan la práctica deportiva, aunado a factores contextuales que tienen que ver con percepción de inseguridad, espacios no apropiados, etc. Lo expuesto plantea la necesidad de involucrar no solo a la familia en esta situación, sino a todos aquellos involucrados en lograr que los niños y las niñas perciban ambientes seguros y motivadores.

Finalmente, se pudo observar que al comparar los argumentos de los padres de niñas y niños activos, versus los padres y madres de las niñas y niños no activos, y, contrario a lo que se hubiese esperado encontrar, no se ubicaron diferencias entre estos dos grupos de actores; es decir, pareciera ser que no existe una relación entre tener o no una hija o hijo activo, en lo que se refiere a las razones que argumentan, como aquellas que limitan o impiden la práctica deportiva en los niños y niñas, en donde se vuelve a encontrar la falta de tiempo y el exceso de responsabilidades como las razones de mayor peso, aunadas al uso de dispositivos electrónicos y falta de motivación y flojera, lo cual confirma lo reportado de manera parcial por otros investigadores (Muñoz-Daw, et al., 2016).

Un elemento interesante está relacionado con las responsabilidades que perciben los niños y las niñas son en realidad responsabilidades propias o son aspectos que están percibiendo de los adultos, lo que entonces permite pensar en la posibilidad de que se esté educando a niños y niñas según el disfrute de su desarrollo (aprender, conocer, anhelar), o si en realidad se les está inculcando desde el hogar la necesidad de una vida presurosa, llena de aspectos por cumplir y realizar.

\section{Conclusiones}

Se requiere contar con mayor evidencia acerca de la situación que ocurre con los hábitos saludables de los niños en México, pues existe una falta de información puntual que conlleva a una deficiencia en las acciones que se toman para intentar fomentar la práctica deportiva, de forma tal que se involucre a los adultos (padres de familia, docentes o entrenadores/as deportivos), cuando existen diversas razones que limitan que los niños y niñas para iniciar o mantener el gusto por el deporte.

Sería recomendable que los padres de familia puedan consultarles a sus hijos sobre sus gustos a la hora de practicar de actividades físico-deportivas, escuchar sus necesidades y, de esta forma, diseñar actividades de manera conjunta, padres-hijos, para que puedan involucrarse en las primeras actividades físico-deportivas de sus hijos, en las cuales se deberán reforzar tanto la participación como los logros, por mínimos que parezcan, e igualmente trabajar los errores como nuevas oportunidades y no como fracasos en estas primeras experiencias.

Uno de los aspectos que resultan fundamentales es considerar la diversión como elemento fundamental en las primeras experiencias del niño y la niña, enfocándose en el juego libre, antes de limitarlo con reglas y 
MHSALUD, ISSN: 1659-097X, 18(2), JULIO-DicIEMBRE, 2021, PP 1-14

Tellez Vasquez, Betancourt Ocampo, Jaimes Reyes, Rubio Sosa, González González

formalidades; en otras palabras, se debe privilegiar los aspectos lúdicos antes que la competencia en lo que respecta a la actividad física.

Otro aspecto fundamental a resaltar es el hecho de que se recomienda evitar que los niños y las niñas practiquen actividades físicas o deportivas que son solo del interés de los padres, pues esto en ocasiones limita la motivación de los niños y las niñas.

Finalmente, sería importante promover que los adultos del contexto cercano a los menores, incluidos los propios padres y madres, sean físicamente activos, ya que no deben olvidar que son modelos para los niños y las niñas y que estos cumplen un papel fundamental en el desarrollo de la población infantil de nuestras sociedades.

\section{Agradecimientos}

El presente estudio se realizó gracias al financiamiento de The Aspen Institute México.

\section{ReFERENCIAS}

American Academy of Pediatrics. (2016). Care of the Young Athlete Patient Education Handouts. https://www.healt hychildren.org/Spanish/healthy-living/fitness/Paginas/overcoming-obstacles-to-physical-activity.aspx

Álvarez, C. (2016). Entendiendo los factores que determinan la actividad física en el entorno escolar desde la perspectiva de los niños y niñas. MHSalud: Revista en Ciencias del Movimiento Humano y Salud, 13(1), 1-17. h ttp://dx.doi.org/10.15359/mhs.13-1.2

Arón, A. y Milicic, N. (1999a). Clima social escolar y desarrollo personal. Un programa de mejoramiento. Editorial Andrés Bello. https://doi.org/10.2307/j.ctt1trkk9z

Arón, A. y Milicic, N. (1999b). Vivir con otros. Programa de desarrollo de habilidades sociales. Editorial Universitaria.

Bornstein, D. B., Beets, M. W., Byun, W., y McIver, K. (2011). Accelerometer-derived physical activity levels of preschoolers: a meta-analysis. Journal of Science and Medicine in Sport, 14(6), 504-511. https://doi.org/10.101 6/j.jsams.2011.05.007

Bronfenbrenner, U. (1979). The ecology of human development. Harvard University Press.

Bronfenbrenner, U. (1989). Ecological system theory. En R. Vasta (Comp.), Annals of childs development. A Research Annual. Six theories of child development: revised formulations and current issues. (Vol. 6, pp. 187- 249). JAI Press.

Cristi-Montero, C., Celis-Morales, C., Ramírez-Campillo, R., Aguilar-Farías, N., Álvarez, C. y Rodríguez-Rodríguez, F. (2015). ¡Sedentarismo e inactividad física no son lo mismo!: Una actualización de conceptos orientada a la prescripción del ejercicio físico para la salud. Revista médica de Chile, 143(8), 1089-1090. https://dx.doi.org/1 0.4067/S0034-98872015000800021

Duverger, M. (1986). Métodos de las Ciencias Sociales. Editorial Ariel.

Encuesta Nacional de Salud y Nutrición. (2016). Informe final de resultados 2016. https://www.gob.mx/cms/upload s/attachment/file/209093/ENSANUT.pdf.

Férnandez-Argëlles, D., y Fernández-Río, J. (2018). Índice de masa corporal, motivos de práctica deportiva extraescolar y modelos familiares en alumnado de $6^{\circ}$ de Educación Primaria. Nutrición Hospitalaria, 35(3), 557-563. https ://doi.org/10.20960/nh.1473

Granda, J., Montilla, M., Barbero, J., Mingorance, A., y Alemany, I. (2010). Frecuencia de práctica y motivos de participación/no participación en actividades físicas en función del género de escolares de 10-12 años de Melilla. Revista Internacional de Ciencias del Deporte, 21(6), 280-296. https://doi.org/10.5332/ricyde2010.02103

Gutiérrez, J., Rivera-Dommarco, J., Shamah-Levy, T., Villalpando-Hernández, S., Franco, A., Cuevas-Nasu, L., Romero-Martínez, M. y Hernández-Ávila, M. (2012) Encuesta Nacional de Salud y Nutrición: Resultados Nacionales. Instituto Nacional de Salud Pública. 
MHSALUD, ISSN: 1659-097X, 18(2), JULIO-DiCIEMBRE, 2021, PP 1-14 Tellez Vasquez, Betancourt Ocampo, Jaimes Reyes, Rubio Sosa, González González

Haugland, S., Wold, B., y Torsheim, T. (2003). Relieving the pressure? The role of physical activity in the relationship between school-related stress and adolescent health complaints. Res Q Exerc Sport, 74(2), 127-135. https://do i.org/10.1080/02701367.2003.10609074

Hernández, M., Rivera, J., Shamah, T., Cuevas, L., Gómez, L., Gaona, E., Romero M., Méndez, I., Saturno, P., Villalpando, S., Guitierrez, J., Ávila, M., Mauricio, E., Martínez, J., y García, D. (2016). Encuesta Nacional de Salud y Nutrición de Medio Camino: Informe final de resultados. Instituto Nacional de Salud Pública.

Instituto Nacional de Salud Pública y Instituto Nacional de Estadística y Geografía. (2018). Encuesta Nacional de Salud y Nutrición: Presentación de resultados. https://ensanut.insp.mx/encuestas/ensanut2018/doctos/inform es/ensanut_2018_presentacion_resultados.pdf

Laing, E., Massoni, J., Nickols-Richardson, S., Modlesky, C., O’Connor, P. \& Lewis, R. (2002). A prospective study of bone mass and body composition in female adolescent gymnasts.J Pediatr, 141(2), 211-216. https://doi.or $\mathrm{g} / 10.1067 / \mathrm{mpd} .2002 .126599$

Martínez, O., Fernández, E. y Camacho, M. (2010). Percepción de dificultades para la práctica de actividad física en chicas adolescentes y su evolución con la edad, Apuntes Educación Física y Deportes, 1(99), 92-99. https://raco. cat/index.php/ApuntsEFD/article/view/249008

Matsudo, S. (2012). Actividad física: Pasaporte para la salud. Revista Médica Clínica Las Condes, 23(3), 209-217. ht tps://doi.org/10.1016/S0716-8640(12)70303-6

Muñoz-Daw, M., Muñóz-Duarte, M., De la Torre-Díaz, M., Hinojos-Seáñez, E. y Pardo-Rentería, J. (2016). Motivos para la práctica de actividad física recreativa e inactividad en la población de Chihuahua (México). Nutrición Clínica y Dietética Hospitalaria, 36(1), 10-16. https://doi.org/10.12873/361 muñozdaw

Organización Mundial de la Salud. (2010). Recomendaciones mundiales sobre la actividad fisica para la salud. https:// apps.who.int/iris/bitstream/handle/10665/44441/9789243599977_spa.pdf

Organización Mundial de la Salud. (2018). Actividad fisica. https://www.who.int/es/news-room/fact-sheets/detail/ physical-activity

Organización Mundial de la Salud. (2019a). Actividad fisica. https://www.who.int/es/news-room/fact-sheets/detail /physical-activity

Organización Mundial de la Salud. (2019b). Estrategia mundial sobre régimen alimentario, actividad física y salud. ht tps://www.who.int/dietphysicalactivity/pa/es/

Organización Mundial de la Salud. (2004c). Estrategia mundial sobre régimen alimentario, actividad fisica y salud. $\mathrm{h}$ ttp://www.who.int/dietphysicalactivity/strategy/eb11344/strategy_spanish_web.pdf

Pietilainen, K., Kaprio, J., Borg, P., Plasqui, G., Yki-Jarvinen, H., Kujala, U., Rose, R.J., Westerterp. K.R. \& Rissanen, A. (2008). Physical inactivity and obesity: a vicious circle. Obesity (Silver Spring), 16(2), 409-414.

Portela, I. y López, A. (2017). Percepción de barreras para la práctica del ejercicio físico en adolescentes gallegos, Revista de Estudios e Investigación en Piscologia y Educación, Extr (14), 174-177. https://doi.org/10.17979/reipe.2017 .0 .14 .2630

Roberts, S. (2000). The role of physical activity in the prevention and treatment of childhood obesity. Pediatr Nurs, 26 (1), 39-41. PMID: 12026314.

Serra, J., Generelo, E. y Zaragoza, J. (2010). Barreras para la realización de actividad física en adolescentes en la provincia Huesca, Revista Internacional de Medicina y Ciencias de la Actividad Fisica y el Deporte, 10(39), 470-482. https ://www.redalyc.org/pdf/542/54223002009.pdf

Schmutz, E., Leeger, C., Radtke, T., Muff, S., Kakebeeke, T., Zysset, A., Messerli, N., Stulb, K., Arhab, A., Meyer, A., Munsch, S., Puder, J., Jenni, O \& Kriemler, S. (2017). Correlates of preschool children's objectively measured physical activity and sedentary behavior: a cross-sectional analysis of the SPLASHY study. International.Journal of Behavioral Nutrition and Physical Activity, 14(1). https://doi.org/10.1186/s12966-016-0456-9

The Aspen Institute (2018). State of Play: Youth Sports Survey. [internet]. http://www.projectplay.us

The Global Observatory for Physical Activity-GoPA. (2016). $1^{\text {st }}$ Physical Activity Almanac. [Internet]. https://indd. adobe.com/view/f8d2c921-4daf-4c96-9eaf-b8fb2c4de615 
MHSALUD, ISSN: 1659-097X, 18(2), JULIO-DiCIEMBRE, 2021, PP 1-14

Tellez Vasquez, Betancourt Ocampo, Jaimes Reyes, Rubio Sosa, González González

Twisk, J., Kemper, H., van Mechelen, W. (2000). Tracking of activity and fitness and the relationship with cardiovascular disease risk factors. Med Sci Sports Exerc, 32(8), 1455-1461. https://doi.org/10.1097/0000576 8-200008000-00014

Vigotsky, L. (1979). El desarrollo de los procesos psicológicos superiores. Editorial Crítica.

World Health Organization. Global (2016) Health Observatory Data Repository. Prevalence of insufficient physical activity among school going adolescents [internet]. https://apps.who.int/gho/data/view.main.2482ADO?lang= en

World Obesity Federation (2019). Atlas of Childhood Obesity. https://data.worldobesity.org/publications/11996-C hildhood-Obesity-Atlas-Report-ART-V2.pdf

Yamamoto-Kimura, L., Alvear-Galindo, M., Morán-Álvarez, C., Acuña-Sánchez, M., Torres-Durán, P., JuárezOropeza, M., Ferreira-Hermosillo, A. y Solís-Díaz, M. (2013). Actividad extraescolar y obesidad en los niños: Influencia del ámbito familiar y del vecindario. Revista Médica del Instituto Mexicano del Seguro Social, 51(4), 378-383. https://doi.org/10.21840/siic/150140 